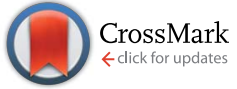

Cite this: DOI: $10.1039 / \mathrm{c} 5 \mathrm{sm} 00022 \mathrm{j}$

Received 5th January 2015

Accepted 16th January 2015

DOI: $10.1039 / c 5 s m 00022 j$

www.rsc.org/softmatter

\section{Searching for line active molecules on biphasic lipid monolayers $\uparrow$}

\begin{abstract}
Andrea Alejandra Bischof, Agustín Mangiarotti and Natalia Wilke*
In membranes with phase coexistence, line tension appears as an important parameter for the determination of the amount of domains, as well as their size and their shape, thus defining the membrane texture. Different molecules have been proposed as "linactants" (i.e. molecules that reduce the line tension, thereby modulating the membrane texture). In this work, we explore the efficiency of different molecules as linactants in monolayers with two coexisting phases of different thicknesses. We tested the linactant ability of a molecule with chains of different saturation degrees, another molecule with different chain lengths and a bulky molecule. In this way, we show in the same system the effect of molecules with chains of different rigidities, with an intrinsic thickness mismatch and with a bulky moiety, thereby analyzing different hypotheses of how a molecule may change the line tension in a monolayer system. Both lipids with different hydrocarbon chains did not act as linactants, while only one of the bulky molecules tested decreased the line tension in the monolayer studied. We conclude that there are no universal rules for the structure of a molecule that enable us to predict that it will behave as a linactant and thus, designing linactants appears to be a difficult task and a challenge for future studies. Furthermore, in regard to the membrane texture, there was no direct influence of the line tension in the distribution of domain sizes.
\end{abstract}

\section{Introduction}

In many model lipid membranes (monolayers and bilayers, free-standing and supported, composed of pure lipids or mixtures of two or more lipids), the coexistence of two-phases has been observed and the distribution of the phases in the membrane plane (texture) has been studied. ${ }^{\mathbf{1 - 1 0}}$ These studies suggest that line tension is a parameter of paramount importance for the observed texture, since it determines the radius of a stable nucleus when the denser phase is generated as well as the rate of nucleation, which in turn regulates the nucleus density and the domain size and shape..$^{2-11}$

It has been proposed that, by analogy with surfactants in $3 \mathrm{D}$ systems, suitably designed line-active compounds, linactants, might be able to create and stabilize 2D molecular nanostructures. ${ }^{11}$ Studies have demonstrated the modification of the "bare" line tension of a system of coexisting phases. Typically, one component in the system is found to partition preferentially

Centro de Investigaciones en Química Biológica de Córdoba (CIQUIBIC), Departamento de Química Biológica, Facultad de Ciencias Químicas, Universidad Nacional de Córdoba, Pabellón Argentina, Ciudad Universitaria, X5000HUA Córdoba, Argentina. E-mail: wilke@mail.fcq.unc.edu.ar; Fax: +54 351 4334171; Tel: +543514334171

$\dagger$ Electronic supplementary information (ESI) available: Movie S1: real time lapse: $1.31 \mathrm{~s}$ (time between frames: $0.082 \mathrm{~s}$ ). Real size: $234.1 \mu \mathrm{m} \times 224 \mu \mathrm{m}$. Movie S2: real time lapse: $2.95 \mathrm{~s}$ (time between frames: $0.082 \mathrm{~s}$ ). Real size: $255.4 \mu \mathrm{m} \times 88.5 \mu \mathrm{m}$. See DOI: $10.1039 / \mathrm{c} 5 \mathrm{sm} 00022 \mathrm{j}$ at phase boundaries. This modification was in some cases caused by impurities within the experimental system. Muller and Gallet $^{\mathbf{1 2}}$ performed experiments with approximately $2 \%$ unlabeled stearic acid impurities that reduced line tension. ${ }^{\mathbf{1 2}}$ Benvegnu and McConnell also observed a decrease in line tension when studying mixtures of DMPC and cholesterol; the impurity cholestonone was found to significantly reduce line tension in the system. ${ }^{\mathbf{1 3}}$

Recently, the idea of actively controlling line tension in a system has become increasingly appealing. One motivation is related to the raft hypothesis in cell membranes. Rafts are described as submicron domains that are enriched in cholesterol and sphingolipids, and it is proposed that they play an important role in cell signaling. The putative size of lipid rafts is in the $10-100 \mathrm{~nm}$ range, ${ }^{14}$ and therefore, a reduction of line tension has been pointed out as a requirement for raft stability and it was hypothesized that some component within the cell membrane reduces the line tension of these rafts naturally, acting as a natural line-active agent. ${ }^{15,16}$

A second motivation is the increasing interest in creating molecular nanostructures on surfaces (e.g., for nanoarray and molecular electronic applications), which requires the presence of stable 2-dimensional nanopatterns. Controlling the size of small nanostructures clearly requires some method to reduce the line tension.

A linactant should consist of three distinct chemical moieties: an anchoring "headgroup" that determines the molecular 
orientation in the membrane, and two other chemical groups, each of which interacts preferentially with one of the coexisting phases. A "perfect linactant" would be a molecule that accumulates at the domain boundary, occupying $100 \%$ of this region.

Trabelsi et al. demonstrated the effectiveness of rationally designed linactant compounds ${ }^{17}$ using mixtures of fatty acids with perfluorated hydrocarbon chains. In more biological-like systems, hybrid lipids (phospholipids with one saturated acyl chain and one unsaturated chain), which are abundant in cell plasma membranes ${ }^{18}$ have been proposed as linactants, ${ }^{\mathbf{1 6 , 1 9 - 2 1}}$ decreasing line tension at membrane domain boundaries and thereby reducing domain sizes.

Quantitatively, the mechanisms of controlling line tension and thus membrane texture have just begun to be explored and there is still no consensus on whether hybrid lipids may act as perfect linactants and, in general, what the chemical requirements are for a surfactant to act as a linactant in a given system.

In this work, we explore the linactant properties of different molecules in monolayers composed of dilauroyl phosphatidylcholine (DLPC) and dihydrocholesterol (dchol, a less hydrolyzable cholesterol analog). ${ }^{22}$ Monolayers of this mixture have been shown to phase-segregate in two 2-dimensional phases which differ in their thicknesses, at low surface pressures. ${ }^{23}$ The mismatch in the height was proposed as an important parameter for the line tension both in monolayers ${ }^{24}$ and in bilayers. ${ }^{25}$

We first checked the line tension reducing ability of the fluorescent probe, which is a required third component in the system because the line tension was determined following the kinetics of the shape relaxation of domains that were previously deformed and the observation was performed using fluorescence microscopy. ${ }^{23}$ The fluorescent moiety of this kind of molecule is bulky and thus may accommodate preferentially in defects such as those present at the domain boundary. Two different fluorescent probes were tested: $\alpha$-phosphatidylethanolamine- $N$-lissamine rhodamine B sulfonyl-ammonium salt, chicken egg-transphosphatidylated (RhoPEegg) and 1,1'-dioctadecyl-3,3,3', $3^{\prime}$-tetramethylindocarbocyanine perchlorate (DiIC18).

Then we tested as a linactant a molecule with chains of different degrees of unsaturation (1-palmitoyl-2-oleoyl-sn-glycero-3-phosphocholine, POPC) and another molecule with different chain lengths (1-palmitoyl-2-lauroyl-sn-glycero-3-phosphocholine, PLPC). In this way, we show in the same system (DLPC/dchol) the effect of molecules with chains of different rigidities, with an intrinsic thickness mismatch and with a bulky moiety and thus analyze three different hypotheses of how a molecule may change the line tension in a monolayer system.

From our results, we conclude that, in agreement with what was found in bilayers by other authors, ${ }^{26}$ hybrid lipids do not act as molecules reducing line tension in all systems. On the other hand, a molecule with hydrocarbon chains of different lengths that may decrease the energy cost of the hydrophobic mismatch caused by the difference in thickness of the coexisting phases appears not to affect line tension. Finally, the fluorescent probe, which is a bulky molecule that may accommodate more easily in defects such as a domain boundary, may or may not act as a linactant, depending on its chemical structure in an unknown way. Thus, the energy decrease derived by the favorable interactions that are generated when these kinds of molecules occupy the interface between an ordered and a disordered region is not always enough to overcome the decrease in entropy derived from such ordered distribution of molecules in the plane of the membrane, and therefore, designing linactants appears to be a difficult task and is still a challenge for future studies.

\section{Materials and methods}

\subsection{Materials}

Dilauroyl phosphatidylcholine (DLPC), dipalmitoyl phosphatidylcholine (DPPC), 1-palmitoyl-2-oleoyl-sn-glycero-3-phosphocholine (POPC), the lipophilic fluorescent probe $\mathrm{L}, \alpha-$ phosphatidylethanolamine- $N$-lissamine rhodamine B sulfonylammonium salt and chicken egg-transphosphatidylated (RhhoPEegg) were purchased from Avanti Polar Lipids. DiIC18 (1,1'-dioctadecyl-3,3,3' , $3^{\prime}$-tetramethylindocarbocyanine perchlorate) was purchased from Molecular Probes and dihydrocholesterol (dchol, a less hydrolyzable cholesterol analog ${ }^{22}$ ) was obtained from Sigma. 1-Palmitoyl-2-lauroyl-sn-glycero-3phosphocholine was a generous gift from Dr Bruno Maggio.

Solvents and chemicals were of the highest commercial purity available. The water used for the subphase was from a Milli-Q system (Millipore), $18 \mathrm{M} \Omega \mathrm{cm}$ and lipid monolayers were prepared and characterized on subphases of $0.15 \mathrm{M} \mathrm{NaCl}$ at 21 $\pm 1{ }^{\circ} \mathrm{C}$.

\subsection{Monolayer observation}

The monolayer was observed while compressed using Fluorescence Microscopy and simultaneously registering the lateral pressure and mean molecular area of the film. The fluorescent probe was incorporated into the lipid solution before spreading. A Langmuir film balance (microthrough, Kibron, Helsinki, Finland) was placed on the stage of an inverted fluorescence microscope (Axiovert 200, Carl Zeiss, Oberkochen, Germany) with a long distance $20 \times$ objective (Zeiss) and images were registered by using a CCD video camera (IxonEM+ model DU897, Andor Technology). The excitation of the fluorescent probes was performed using a continuous solid state laser (TEM00, $532 \mathrm{~nm}$ up to $200 \mathrm{~mW}$, Roithner Lasertech) and for the detection, a rhodamine emission filter was added to the optical path. In all experiments, the fluorescent probe concentration in the denser phase was lower, and thus this phase appeared darker in the micrographs.

In order to test the presence of a concentration gradient of DiIC18 at the domain boundaries, monolayers of DLPC/dchol (3:1) and DLPC/DPPC (2:3) with very low proportions of the probe $(0.02 \%)$ were prepared and transferred to a hydrophilic glass slide by the Langmuir-Blodgett technique ${ }^{\mathbf{1 0}}$ at 4 and 30 $\mathrm{mN} \mathrm{m}^{-1}$, respectively. The immobilized monolayers were then observed under the microscope using long exposure times (1 min) with a $100 \times$ objective. 


\subsection{Deformation of the domain shapes and determination} of the line tension

If the dipole density of the domains is different from that of the continuous phase, a nonhomogeneous electric field will generate a net force on the domains and cause their migration from or to the region under the upper electrode $e^{27,28}$ and subsequently lead to their deformation. ${ }^{23}$ The experimental setup for applying an electrostatic field to the lipid monolayer was carried out as described previously. ${ }^{27}$ Briefly, a metal wire was held at $200 \mu \mathrm{m}$ above the subphase, a second electrode was placed in the subphase and a potential difference was applied between

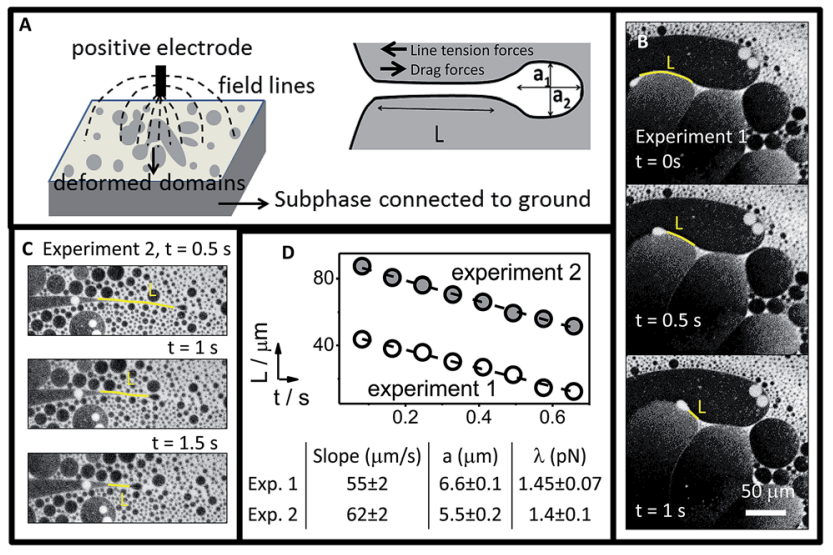

Fig. 1 Representative experiments for the determination of the line tension. (A) Scheme of the experimental setup. The involved forces are indicated qualitatively in the figure. ( $\mathrm{B}$ and $\mathrm{C}$ ) Monolayers composed of DLPC/dchol (3: 1) with $0.5 \mathrm{~mol} \%$ of RhoPEegg at $4 \mathrm{mN} \mathrm{m}^{-1}$ on $0.15 \mathrm{M}$ $\mathrm{NaCl}$. In experiment 1 the stripe is composed of the DLPC enriched phase and in experiment 2 of the dchol enriched phase. The determined length $(L)$ is highlighted in yellow. (D) Length of the stripes ( $L$, yellow lines) in experiments 1 and 2 as a function of time and the corresponding values determined from the experiments shown in $B$ and $\mathrm{C}$. the electrodes (see Fig. 1A). The upper electrode was charged by applying potentials of up to $300 \mathrm{~V}$ with respect to the subphase electrode. Some very long domain strips are generated that break at random and if the field is turned off at this stage, the relaxation of the strip can be followed over time. ${ }^{23}$ Fig. $1 \mathrm{~B}$ and $\mathrm{C}$ show typical experiments and the corresponding movies can be found in the ESI (Movies S1 and S2 $\dagger$ ). The driving force of the relaxation of the strip can be thought of as that of a spring whose strength depends on the line tension that moves the head of the strip closer to the domain and the friction that opposes the movement (see scheme in Fig. 1A). If the head of the strip moves at a constant velocity, the forces acting on the system are balanced during this process. ${ }^{23,29}$

The electrostatic repulsion due to the difference in dipole density of each phase can be neglected since the velocity of the stripe retraction is constant; ${ }^{23}$ additionally, the results obtained with a stripe formed with the phase enriched in the fluorescent probe lead to the same result as that formed with the other phase (compare experiments 1 with 2 in Fig. 1). Under these conditions, it was previously shown that line tension $(\lambda)$ can be calculated as

$$
\lambda=4 \eta a \frac{\mathrm{d} L}{\mathrm{~d} t}
$$

here $\eta$ is the subphase viscosity $\left(1 \times 10^{-3} \mathrm{~N} \mathrm{~s} \mathrm{~m}^{-1}\right), a$ is the radius of the head of the stripe and can be approximated as $\sqrt{a_{1} \times a_{2}} / 2$ since it is usually not a circle but an ellipse, and $L$ is the length of the stripe (see Fig. 1A). Note that in the study by Bischof et al., ${ }^{23}$ the equation presents a typographical error (a value of $\eta$ of $1 \times 10^{-2} \mathrm{~N} \mathrm{~s} \mathrm{~m}^{-1}$ was written instead of $1 \times 10^{-3} \mathrm{~N} \mathrm{~s} \mathrm{~m}^{-1}$ ).

Line tension was determined using this procedure at $4 \mathrm{mN} \mathrm{m}^{-1}$ for monolayers on $0.15 \mathrm{M} \mathrm{NaCl}$ at $21 \pm 1{ }^{\circ} \mathrm{C}$ and an example of the procedure is shown in Fig. 1 along with the calculated values (Fig. 1D). In a working day, a mixture of dchol with the studied phospholipid and 1\% of RhoPEegg was prepared and the line tension of at least 3 videos recorded from

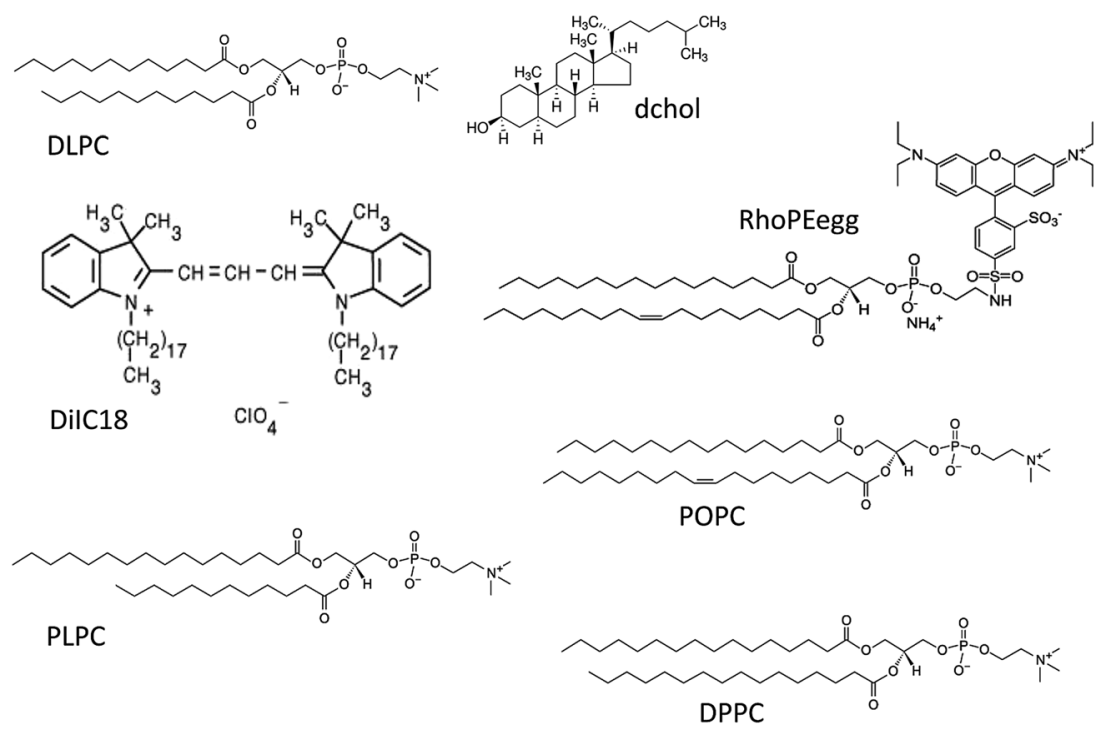

Fig. 2 Chemical structures of the surfactants used in this study. 

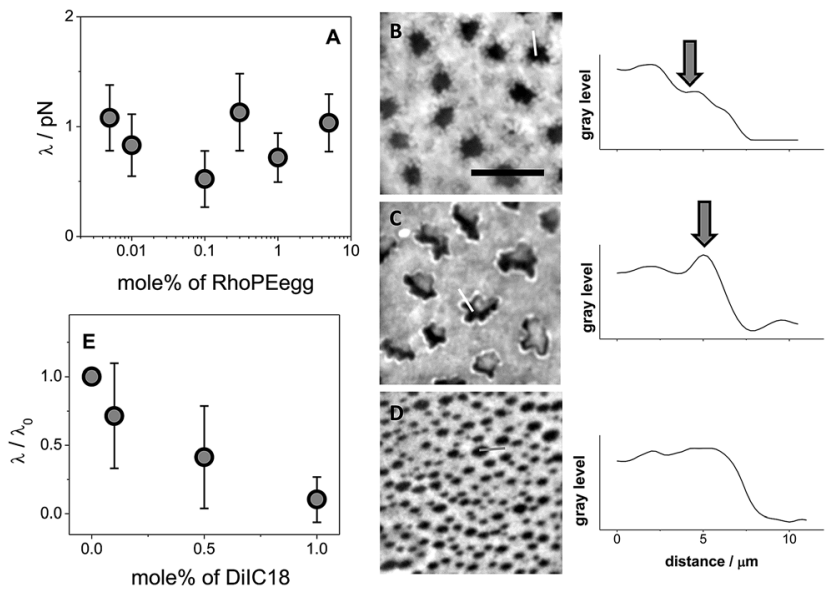

Fig. 3 (A) Line tension values for monolayers composed of DLPC/ dchol $(3: 1)$ and increasing proportions of the fluorescent probe RhoPEegg at $4 \mathrm{mN} \mathrm{m}^{-1}, 21{ }^{\circ} \mathrm{C}$ and on subphases of $0.15 \mathrm{M} \mathrm{NaCl}$. (B) Image of a monolayer composed of DLPC/DPPC (2 : 3) with $0.02 \%$ of RhoPEegg transferred to the glass at $30 \mathrm{mN} \mathrm{m}^{-1}$. The bar corresponds to $25 \mu \mathrm{m}$. Right: plot of the level of gray vs. the distance along the white line in the image. (C) Image of a monolayer composed of DLPC/DPPC $(2: 3)$ with $0.02 \%$ of DilC18 transferred to the glass at $30 \mathrm{mN} \mathrm{m}^{-1}$, the same scale as in B. Right: plot of the level of gray $v$ s. the distance along the white line in the image. (D) Image of a monolayer composed of DLPC/dchol $(3: 1)$ with $0.02 \%$ of DilC18 transferred to the glass at 4 $\mathrm{mN} \mathrm{m}{ }^{-1}$, the same scale as in B. Right: plot of the level of gray vs. the distance along the gray line in the image. (E) Normalized line tension for monolayers composed of DLPC/dchol (3:1) with 0.5 mol\% of RhoPEegg and increasing proportions of the fluorescent probe DilC18 at $4 \mathrm{mN} \mathrm{m}^{-1}, 21^{\circ} \mathrm{C}$ and on subphases of $0.15 \mathrm{M} \mathrm{NaCl}$.

one monolayer of this mixture was determined. This was considered the control experiment. Then, different amounts of POPC, DiIC18 or PLPC were added to the previous mixture, a monolayer was prepared and the line tension of at least 3 videos recorded was determined. The whole procedure was repeated 3 times and the obtained line tensions were normalized by the line tension of the corresponding control experiment. Each series of experiments showed the same tendency and the results obtained with one of these series are presented (Fig. 3 and 4).

Additionally, in order to increase the data statistics, control experiments and experiments with $1 \%$ of the putative linactive molecule were measured on the same day. For these
Table 1 Values for the line tension determined as indicated in the Experimental section. The error corresponds to the standard deviation of 8 independent experiments

\begin{tabular}{lll}
\hline System & Control $(\mathrm{pN})$ & $\begin{array}{l}1 \% \text { of the tested } \\
\text { molecule }(\mathrm{pN})\end{array}$ \\
\hline DLPC/dchol/PLPC & $1.0 \pm 0.2$ & $0.9 \pm 0.4$ \\
DLPC/dchol/POPC & $1.0 \pm 0.2$ & $1.1 \pm 0.5$ \\
DLPC/dchol/DiIC18 & $1.3 \pm 0.4$ & $0.1 \pm 0.2$ \\
DPPC/dchol/POPC & $0.7 \pm 0.2$ & $0.6 \pm 0.4$
\end{tabular}

experiments, two monolayers of each composition were prepared and 8 experiments were analyzed for each system. The results are summarized in Table 1 , along with the standard error (calculated from the average of the 8 data).

The determination of the line tension with a low amount of RhoPEegg was performed using the excitation laser at the maximal intensity, and turning it on only during data acquisition to minimize photo-bleaching. The determination of the line tension under these conditions was repeated at least 5 times in order to obtain an accurate value of $\lambda$.

\section{Results and discussion}

\subsection{Line tension for the ternary mixtures analyzed}

The phase behavior of mixtures of DLPC and dchol follows the general trend reported for mixtures of phospholipids and cholesterol. ${ }^{30}$ At the working temperature $\left(21 \pm 1^{\circ} \mathrm{C}\right)$, the system showed phase segregation for the molar fraction of dchol higher than 0.1 . The range $0.1<x_{\text {dchol }}<0.3$ and surface pressures lower than about $14 \mathrm{mN} \mathrm{m}^{-1}$ (ref. 23) correspond to the $\alpha$ region of the phase diagram: ${ }^{30}$ depending on the lipid proportion, rounded domains enriched in dchol that exclude the fluorescent probe are inserted in a continuous phase enriched in DLPC, or the opposite, domains enriched in DLPC are inserted in a phase enriched in dchol. In both cases, domains are stable in time and do not fuse. The line tension was determined in monolayers composed of DLPC/dchol (3:1), i.e. in the $\alpha$ region of the phase diagram. The general features and the mixing pressure $\left(14 \mathrm{mN} \mathrm{m}^{-1}\right)$ did not change in the presence of different amounts of RhoPEegg, DiIC18, PLPC or

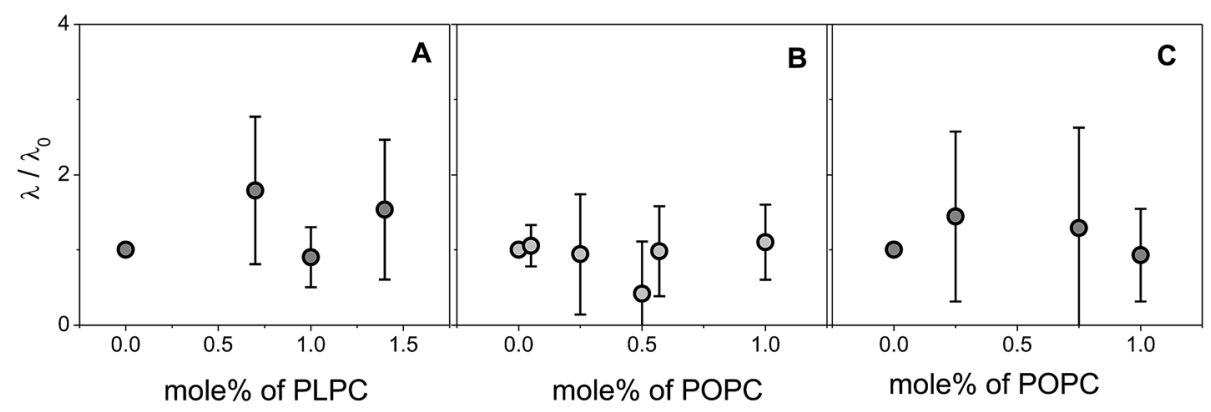

Fig. 4 Normalized line tension for monolayers composed of DLPC/dchol $(3: 1)$ with $0.5 \mathrm{~mol} \%$ of RhoPEegg and increasing proportions of PLPC (A) or POPC (B) at $4 \mathrm{mN} \mathrm{m}^{-1}, 21^{\circ} \mathrm{C}$ and on subphases of $0.15 \mathrm{M} \mathrm{NaCl}$. (C) Normalized line tension for monolayers composed of DPPC/dchol (3 : 1) with $0.5 \mathrm{~mol} \%$ of RhoPEegg and increasing proportions of POPC at $4 \mathrm{mN} \mathrm{m}^{-1}, 21^{\circ} \mathrm{C}$ and on subphases of $0.15 \mathrm{M} \mathrm{NaCl}$. 
POPC. The distribution of domain sizes remains constant in time, at least during the experiment ( $1-2$ hours) for all experiments.

The line tension in monolayers composed of mixtures of DLPC and dchol was determined experimentally as briefly explained in the Experimental section and detailed in the study by Bischof et al. ${ }^{23}$ As fluorescence microscopy was used for the monolayer observation, a fluorescent probe had to be added to the lipid mixture in all cases.

In order to ensure that the possible effects on line tension detected when a new component was added to the mixture were not related to changes in properties of the bulk phases, very low amounts of the putative linactants were used. A rough estimation of which concentration should be evaluated was performed considering an "ideal linactant behavior", i.e. that $100 \%$ of the linactant molecules accumulate at the domain boundaries. The estimation of the amount of linactant necessary for the saturation of the domain borders was performed as follows: the average domain size was $15 \mu^{2}$, leading to an average domain perimeter of $13 \mu \mathrm{m}$, whereas the average area occupied by a domain was $\sim 100 \mu^{2}$. Considering a probe with a mean molecular area of $1 \mathrm{~nm}^{2}$ (mean molecular diameter of $1 \times 10^{-3}$ $\mu \mathrm{m}), 10 \mu \mathrm{m}$ of border saturates with $\sim 1 \times 10^{4}$ molecules of the probe. As already mentioned, $\sim 10 \mu \mathrm{m}$ of border was in an average region of $100 \mu^{2}$, the amount of molecules in this region being $100 \mu \mathrm{m}^{2} / 0.8 \mathrm{~nm}^{2} \sim 1 \times 10^{8}$ molecules. Thus, $0.01 \%$ in moles of each putative linactant would saturate the domain borders. This is a lower limit; in a real situation, a higher molar fraction of molecules is expected to saturate the domain borders, since entropy would lead to a non-complete accumulation in the domain border, and therefore, a Boltzmann distribution with preference in the lineal interface between the phases is expected.

3.1.1 Line tension in the presence of a bulky molecule. The fluorescent probe is usually a lipid-like molecule with a fluorophore group attached to the polar head-group of the molecule. This fluorophore is a bulky moiety, and thus the pure probe usually forms films with expanded behavior at the airwater interfaces, and when present in a mixture, it concentrates in the less packed regions of the monolayer. It may also happen that, being a bulky molecule, it accommodates in defects in the monolayer.

The boundary of a domain may be considered as a disordered region, with more defects than the bulk of each coexisting phase. The existence of defects in domain boundaries has been proposed initially to explain the increase in lipid membrane conductivity during phase transition. ${ }^{31}$ In that work, the authors assigned a higher permeability to the domain borders compared to those of each bulk phase, due to the high defect density in this interfacial region which was proposed to lead to bad packing and leakiness. Afterwards, accumulation of proteins and peptides at domain borders was reported for several systems and the presence of defects in those boundaries was proposed as a possible explanation for this observation. ${ }^{32-34}$ Additionally, the accumulation of proteins at the boundary was predicted assuming the monolayer as a lattice composed of sites occupied by lipids, proteins and vacancies: ${ }^{36}$ when the proteins are energetically indifferent to the density of amphiphiles, they will localize at the line boundary between the coexisting phases as a consequence of entropic forces. This model is not only valid for proteins but in general for molecules occupying an area larger than the lipid molecules and for all kinds of coexisting phases. This model also predicts a reduction in the line tension caused by the accumulation of the bulky molecules at the domain boundary.

Taking these considerations into account, the effect of the fluorescent probe in the line tension of DLPC/dchol monolayers $(3: 1)$ was tested. First, the probe used in the study by Bischof et al. ${ }^{23}$ i.e. RhoPEegg, was explored (see the chemical structure in Fig. 2).

The minimal amount of the fluorescent probe that allowed us to observe the kinetic evolution of the domain shapes, and thereby to perform the experiment, was $0.005 \%$ in moles. For that, the excitation laser was set at the maximal intensity, and was turned on only during data acquisition to avoid photobleaching. The determination of the line tension under these conditions was repeated at least 5 times in order to obtain an accurate value of $\lambda$. Fig. 3A shows the value of the line tension of DLPC/dchol mixtures at $4 \mathrm{mN} \mathrm{m}^{-1}$ for increasing values of RhoPEegg, and it can be concluded that this probe does not affect the value of $\lambda$ in biphasic monolayers of DLPC and dchol.

Previous experiments in our laboratory had led to the suspicion that this was not the case for DiIC18 (see the chemical structure in Fig. 2). This molecule was used as a fluorescently labeled probe for the detection of phase coexistence in monolayers composed of the lipids extracted from myelin of bovine brain and myelin basic protein ${ }^{22}$ and it was found that the presence of diIC18 decreased the lateral pressure at which the domains became unstable and fused. In this complex mixture, phase segregation implies also separation of charge since the cationic protein segregates to a different phase than the anionic lipids. Since DiIC18 is a cationic amphiphile, it was proposed that the effect found in monolayers of myelin lipids was related to an electrostatic effect of the probe in each coexisting phase. However, the decrease in the mixing lateral pressure promoted by DiIC18 may also be related to the decrease in line tension, which leads to a concomitant decrease in the domain stability. Additionally, we also noticed that in monolayers composed of DPPC/DLPC (2:3), which present liquid condensed-liquid expanded phase coexistence, DiIC18 (and not RhoPEegg) accumulated at the domain borders. This system forms domains with highly branched shapes which were previously observed using fluorescence microscopy (with RhoPEegg) and Brewster Angle Microscopy (in the absence of a fluorescent probe). ${ }^{10}$ Some domain branches are below the optical resolution and thus, domains appeared blurred, with a halo of a level of the gray intermediate between that of the domain and that of the continuous phase (see Fig. 3B). At the right side of the image, a plot of the gray level $v s$. distance along the white line (inserted in the image) is shown. The arrow indicates the domain border, where an intermediate gray level is observed. The image was measured with a $100 \times$ objective, with $0.02 \%$ RhoPEegg and the lipid proportion was DLPC/DPPC $2: 3$ (transferred to glass at $30 \mathrm{mN} \mathrm{m}^{-1}$ ). If DiIC18 is used instead of 
RhoPEegg (also $0.02 \%$ ), the obtained images showed also a gray halo, but in this case it was brighter than the domain and the continuous phase (Fig. 3C and the corresponding plot of gray level vs. distance). In this system, the accumulation of the fluorescent probe at the domain border could be detected because domains were branched and thus, the region of the image that corresponded to border was thick. The branches appeared blurry because of the low resolution of the technique.

Interestingly, Crane et al. ${ }^{37}$ observed a similar effect using DiIC18 as the fluorescent probe in mixtures of phospholipids, sphingomyelin and cholesterol, although they did not discuss it: in the image corresponding to Fig. 3E of ref. 37, a light gray halo is observed for supported bilayers with $5 \%$ of cholesterol when DiIC18 is used as the fluorescent probe and this halo is absent in bilayers with other four different probes. The images that correspond to different cholesterol proportions do not show this halo, but the absence of a brighter region in the surroundings of the domain does not imply the absence of accumulation of the fluorescent molecule at the domain border since the resolution of optical microscopy is in the $\mu \mathrm{m}$ range. Each ring of molecules around the domain would be about 1 $\mathrm{nm}$ thickness and thus, only when more than 1000 rings of molecules are accumulated it will be possible to observe them by optical microscopy. Therefore, the absence of a light gray halo does not indicate that there is no accumulation of the probe at the domain boundary.

In monolayers of DLPC, dchol and DiIC18, we did not observe a brighter region around the domains. Fig. 3D shows a monolayer composed of DLPC/dchol (3:1) with $0.02 \%$ of DiIC18 transferred at $4 \mathrm{mN} \mathrm{m}^{-1}$ onto the glass. The shapes of the domains were elongated instead of circular as a consequence of the transfer process, since $4 \mathrm{mN} \mathrm{m}^{-1}$ is a very low surface pressure and under these conditions the transfer is not so good. As already discussed, the absence of a light gray halo in transferred films does not indicate that there is no accumulation of the probe at the domain boundary, because it may occur and the technique is failing to detect it.

With this in mind, monolayers of DLPC/dchol $(3: 1)$ with 0.5 mol\% of RhoPEegg were prepared with increasing amounts of DiIC18 and the line tension of these monolayers was tested. The results are shown in Fig. 3B and Table 1; the values of the line tension in the presence of DiIC18 were normalized by the value in the absence of this molecule. A ten-fold decrease of the original value was observed in monolayers with $1 \mathrm{~mol} \%$ of DiIC18, in agreement with the detected accumulation of the probe at the domain boundary in a different system by optical microscopy.

As can be observed in the chemical structure of the molecules shown in Fig. 2, the fluorescent moiety of DiIC18 is bulkier and is closer to the hydrocarbon chains than that of RhoPEegg, and therefore DiIC18 may induce more disorder in the monolayer structure than RhoPEegg, leading to a preference of DiIC18 but not of RhoPEegg at the domain borders, which are already disordered regions. ${ }^{31-37}$

3.1.2 Line tension in the presence of phospholipids with different hydrocarbon chains. Subsequently, the effect on $\lambda$ of two lipids with different hydrocarbon chains was tested in monolayers composed of DLPC/dchol (3:1) with 0.5 mol\% of RhoPEegg. Low amounts of PLPC (with chains $16: 0$ and $12: 0$ ) or POPC (with chains 18:1 and 16:0) were added to the lipid mixture and the line tension of the system was determined.

PLPC was used considering that it contains a hydrocarbon chain that is longer than the other (see chemical structures in Fig. 2), and thus the molecule bears an intrinsic hydrophobic mismatch. It has been proposed in monolayers ${ }^{24}$ and in bilayers $^{25}$ that line tension increases with the hydrophobic mismatch derived from the difference in thickness of the coexisting phases. Therefore, our hypothesis was that a molecule with an intrinsic hydrophobic mismatch may accumulate at the domain borders, decreasing the energy cost related to the mismatch in phase thicknesses.

It was previously reported that in the DLPC/dchol mixture, the thickness of the DLPC-enriched phase is $1.3 \pm 0.1 \mathrm{~nm}$ and that of the dchol-enriched phase is $1.6 \pm 0.1 \mathrm{~nm} .{ }^{23}$ Therefore, at the border of the domain a height mismatch of $0.3 \pm 0.2 \mathrm{~nm}$ has to be overcome. According to Israelachvili, ${ }^{38}$ the length difference between a fully stretched hydrocarbon chain of $16 \mathrm{C}$ and another of $12 \mathrm{C}$ is $0.5 \mathrm{~nm}$, and thus PLPC may accommodate at the domain border as a consequence of the different length of its hydrocarbon chain when fully stretched, thereby decreasing the geometrical tensions generated at the domain border due to the height mismatch. Fig. 4A shows the line tension for a system composed of DLPC/dchol (3 : 1, with $0.5 \mathrm{~mol} \%$ of RhoPEegg), with increasing amounts of PLPC normalized by the $\lambda$ value in the absence of PLPC for a series of experiments (see Experimental section) and Table 1 shows the values for the line tension in the absence and in the presence of 1\% PLPC (average of 8 experiments in two different monolayers). Notwithstanding our hypothesis, the results shown in Fig. 4 and in Table 1 indicate that PLPC did not modify the line tension of the system (within an error of $\pm 0.4 \mathrm{pN}$ ), suggesting that the decrease in energy gained with the PLPC molecules at the domain border is not enough to pay the entropy cost for such ordered distribution of molecules.

POPC (16:0 and $18: 1$ ) was used as a typical hybrid lipid with a saturated and an unsaturated hydrocarbon chain. Hybrid lipids are proposed to decrease line tension when phase coexistence between an ordered and a less ordered phase coexists. ${ }^{\mathbf{1 6}}$ Disorder may arise due to permanent double bonds in an unsaturated chain or due to a temporary gauche configuration of the saturated lipid. In mixtures of DLPC and dchol, the phase enriched in cholesterol is expected to be more ordered and thus, the gauche configurations are less probable, and therefore, POPC may stabilize the domain border as a consequence of accumulation of these molecules in the interfacial region. Hybrid lipids have been shown to decrease ${ }^{16,19-21}$ or not to alter the line tension in different lipid mixtures.

In monolayers composed of DLPC/dchol (3:1) with 0.5 mol\% of RhoPEegg, we found that line tension remained constant within experimental error when the amount of POPC increased (Fig. 4B and Table 1), indicating that this molecule did not act as a linactant in this case. It may be possible that POPC, while not acting as linactant in the DLPC/dchol mixture, decreases the line tension in other systems with different 


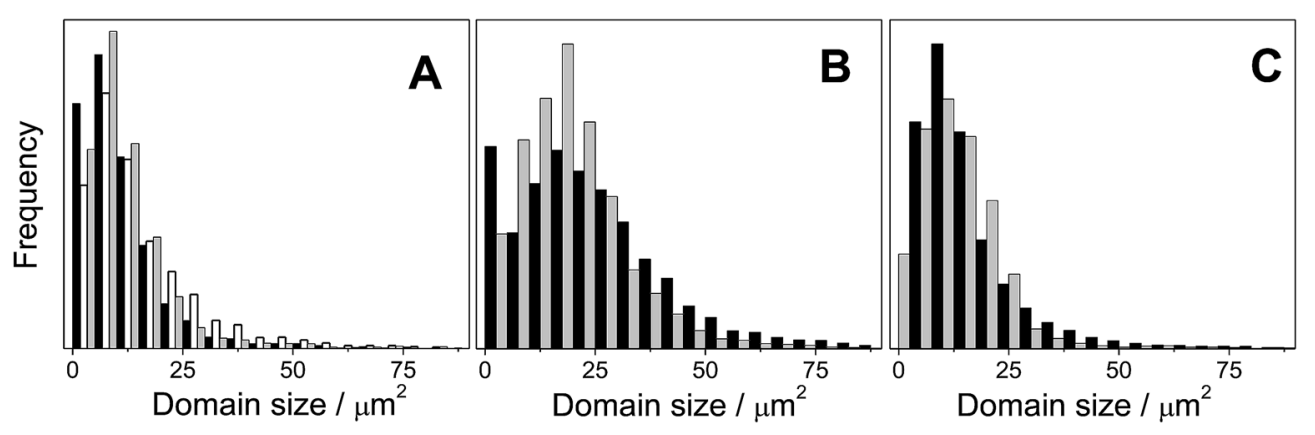

Fig. 5 Distribution of the domain sizes obtained for 50 images of $200 \times 200 \mu \mathrm{m}$ (about 400 domains in each frame) at $4 \mathrm{mN} \mathrm{m}{ }^{-1}, 21{ }^{\circ} \mathrm{C}$ and on subphases of $0.15 \mathrm{M} \mathrm{NaCl}$. (A) Monolayers composed of DLPC/dchol (3:1) with 0.5 mol\% of RhoPEegg (black bars); the same monolayer with $0.5 \%$ of POPC (white bars) or $0.7 \%$ of PLPC (gray bars). (B) Monolayers composed of DPPC/dchol (3: 1 ) with 0.5 mol\% of RhoPEegg (black bars); the same monolayer with $0.75 \%$ of POPC (gray bars). (C) Monolayers composed of DLPC/dchol ( $3: 1)$ with 0.5 mol\% of RhoPEegg (black bars); the same monolayer with $1 \%$ of DilC18 (gray bars).

properties at the domain boundary. Therefore, we examined the effect of POPC in mixtures of DPPC with dchol $(3: 1)$. This composition corresponds to the $\alpha$ region of the phase diagram of the mixture, as for the case of DLPC/dchol $(3: 1)$ but for the mixture of DPPC/dchol the corresponding demixing pressure is $16 \mathrm{mN} \mathrm{m}^{-1} .^{23}$ This value does not change in the presence of the low amounts of POPC used here. As shown in Fig. 4C and Table 1 , no effect on line tension as a consequence of the presence of POPC was found in this system either.

Our results imply that the presence of a saturated and an unsaturated chain in a lipid does not necessarily lead to a decrease in the line tension in a biphasic lipid monolayer. As discussed for molecules with different chain lengths, this suggests that in spite of reducing the energetic cost of the interface, the energy gained by the presence of POPC in the interface is not enough to pay the entropic cost of this ordered distribution of molecules.

The constancy in the line tension in the tested mixed monolayers may be a consequence of the extremely simply system under study, and of the absence of unsaturated lipids. However, we have previously determined the line tension in monolayers composed of myelin lipids purified from bovine spinal cord, a system that contains the complex lipid composition of the cell membrane, and a line tension in the range of 0.65 and $0.95 \mathrm{pN}$ depending on subphase conditions was determined. ${ }^{39}$ Those results and the results shown here suggest that the effect of hybrid lipids on the line tension in monolayers should be revised.

\subsection{Domain size distribution}

In this section, the effect of the presence of a low amount of a new component on the domain size distribution was analyzed. It has been proposed that a low amount of hybrid lipids in particular and linactants in general affect the sizes of domains in membranes, ${ }^{\mathbf{1 5 , 1 6 , 1 9 , 2 4 , 4 0}}$ and therefore this possibility was tested here. Usually, domains in a membrane are polydisperse and the distribution of domain sizes in a monolayer has been correlated with the line tension value. ${ }^{24,41}$ Fig. 5 shows the domain size distribution before the electric field was applied to the monolayers. As shown in Fig. 5A and B, the constancy in line tension values found in this work for both systems in the presence of RhoPEegg, PLPC and POPC correlated with constancy in the texture of the films. However, the domain sizes in monolayers in the presence of DiIC18 were similar to monolayers in their absence, as shown in Fig. 5C.

The equilibrium domain size for circular and isolated liquid domains $\left(R_{\mathrm{eq}}\right)$ depends on the ratio of the line tension to the square of difference in dipole density $\mu^{\mathbf{1}}$ according to:

$$
R_{\text {eq }}=5 \Delta \exp \left(\lambda / \mu^{2}\right)
$$

here, $\Delta$ is a cut off parameter of the order of magnitude of the separation of molecular dipoles ${ }^{1}$ and $\mu^{2}$ can be obtained from surface potential experiments, if $V_{\mathrm{d}}$ (the surface potential of the domain) and $V_{\mathrm{s}}$ (the surface potential of the surroundings) are known using eqn (3): ${ }^{42}$

$$
\mu^{2}=\frac{\varepsilon_{0}}{2 \pi}\left(V_{\mathrm{d}}-V_{\mathrm{s}}\right)^{2}
$$

Domains that are larger than $R_{\text {eq }}$ are not circular but elongated. Eqn (2) indicates that a change in line tension (with $\mu$ constant) leads to a change in $R_{\text {eq }}$. As the domains were circular in all the systems analyzed, $R_{\text {eq }}$ must be larger than the larger observed domain, which was $\sim 100 \mu \mathrm{m}^{2}$ (see Fig. 5). In other words, $\quad R_{\text {eq }} \geq \sqrt{100 \mu \mathrm{m}^{2} / \pi} \cong 6 \mu \mathrm{m}$, otherwise the larger domains would be elongated instead of circular. Assuming $\Delta=$ $1 \mathrm{~nm}$ (ref. 43) and using the lower value determined for $\lambda(0.1$ $\mathrm{pN}$ ), a maximum value for $\mu^{2}$ of $10^{-14} \mathrm{~N}$ (and thus $\Delta V \leq 0.1 \mathrm{~V}$ ) was calculated. This value for $\Delta V$ is in agreement with the experimental values. For example, a value of $0.1 \mathrm{~V}$ was reported for monolayers composed of DMPC and cholesterol (or dchol). ${ }^{\mathbf{4 4}}$

According to McConnell, ${ }^{1}$ the energy of an isolated domain with radius $R$ and area $A_{\mathrm{d}}$ can be calculated from:

$$
E(R)=\frac{2 A_{\mathrm{d}}}{R} \mu^{2}\left(\ln \frac{R_{\mathrm{eq}}}{R}-1\right)
$$

This equation assumes that $E$ depends solely on the electrostatic repulsion and the line tension. In the monolayers studied here, domains are not isolated but the area fraction 
covered by domains is very low (0.15) and thus domains can be treated as isolated. For an average domain area $A_{\mathrm{d}}=15 \mu \mathrm{m}^{2}$ and $R_{\text {eq }}=6 \mu \mathrm{m}, E(2 \mu \mathrm{m})=10^{-8} \mathrm{~J}$ and $E\left(R_{\text {eq }}\right)=-10^{-8} \mathrm{~J}$, with a corresponding thermal energy $k_{\mathrm{B}} T n=10^{-14} \mathrm{~J}\left(n=10^{7}\right.$, the amount of lipids in the domain). Assuming an equilibrium domain size distribution (Boltzmann distribution) and neglecting the contribution of entropy to the total energy, these results imply that the presence of domains with $R=2 \mu \mathrm{m}$ would be very unlikely; however, $\sim 2 \mu \mathrm{m}$ corresponds to the most probable domain size (see the histograms in Fig. 5).

This observation concerning the equilibrium sizes of lipid domains has been previously reported and discussed. ${ }^{1,45-48} \mathrm{~A}$ thermodynamic treatment of the equilibrium sizes of lipid domains was performed by McConnell and it was shown that the broad distribution of sizes is not compatible with thermodynamic but to metastable equilibrium sizes. ${ }^{48}$ Later, it was shown that in certain lipid mixtures, the rate of size equilibration is exceedingly small, thus accounting for the experimental results. ${ }^{43}$ In that work, it was shown that the equilibration rate in binary mixtures of cholesterol and phosphatidylcholine is remarkably slow, the radius of a typical $20 \mu \mathrm{m}$ diameter domain changing by as little as a part in a million per second. Under these circumstances, equilibration times of the order of days or weeks are expected, but even with such long times, the final state reached by the monolayer will be a state of metastable equilibrium, rather than true equilibrium.

We therefore conclude that the monolayers studied here are composed of domains that are kinetically trapped in out-ofequilibrium sizes (as previously reported for mixtures of DMPC and $\mathrm{dchol}^{\mathbf{4 3}}$ ), with an equilibrium radius for shape transition (from circular to elongated shapes) larger than $6 \mu \mathrm{m}$, and with surface potential differences of the order of $10^{2} \mathrm{mV}$.

Since the distribution of domain sizes is not an equilibrium property, it does not necessarily change as a result of a change in $\lambda$. Furthermore, the histograms shown in Fig. 5 suggest that $\lambda$ is not an important factor for the determination of the domain sizes in the studied monolayers.

This behavior is probably the most common case in monolayers with liquid-liquid coexistence and thus, assuming an equilibrium domain size distribution in order to obtain equilibrium parameters (as previously reported ${ }^{\mathbf{2 4 , 4 1}}$ ) would not be correct in most cases. The monolayer texture may lead to equilibrium parameters however, if the experimental equilibrium radius is obtained from the size of domains that undergo a shape transition from circular to elongated domains, as performed before succesfully. ${ }^{49-51}$

Regarding the probability of existence of domains with sizes in the nanometer range, the classical theory of nucleation predicts that the critical size for a stable nucleus is $r_{\mathrm{c}}=\lambda(\mathrm{MMA})$ $\times T_{\mathrm{m}} /(\Delta H \times \Delta T)$, MMA being the mean molecular area of the lipid in the domain and $T_{\mathrm{m}}$ the melting temperature. Considering $\Delta H=100 \mathrm{~kJ} \mathrm{~mol}^{-1}, T_{\mathrm{m}}=300 \mathrm{~K}, \Delta T=10 \mathrm{~K}, \mathrm{MMA}=1 \mathrm{~nm}^{2}$ and $r_{\mathrm{c}}=10 \mathrm{~nm}, \lambda$ is in the order of the $\mathrm{pN}$. In other words, nanodomains may form with line tensions of the order of the $\mathrm{pN},{ }^{52}$ which is the range of values found here and in monolayers composed of lipids purified from myelin. ${ }^{39}$ Furthermore, nanosized domains have been observed in several systems in the absence of putative linactants, coexisting in some proportions with micron-sized domains, for example in supported bilayers composed of DPPC/DLPC/chol using $\mathrm{AFM}^{53}$ and in GUVs composed of DSPC/DLPC/DOPC/chol using FRET and SANS. ${ }^{54}$

Concerning the thermodynamic stability of nano-sized domains, fluctuations in monolayer density can be estimated treating a portion of the monolayer as an open system in equilibrium with the rest of the monolayer which serves as a reservoir, as done by Blank. ${ }^{55}$ If $\bar{n}$ is the mean number of molecules in the area under consideration $(A)$, then the actual number, $n$, at any given time fluctuates according to the thermal energy and to the compressibility modulus of the monolayer $(\kappa)$ as:

$$
\frac{\overline{n-\bar{n}}}{\bar{n}}=\sqrt{\frac{k_{\mathrm{B}} T}{A \kappa}}
$$

If the area under consideration is the average domain area $\overline{A_{\mathrm{d}}}$, then $n=A_{\mathrm{d}} /$ MMA and $\bar{n}=\overline{A_{\mathrm{d}}} /$ MMA. Then, fluctuations in the instantaneous domain area $A_{\mathrm{d}}$ with respect to the average value $\overline{A_{\mathrm{d}}}$ can be estimated as:

$$
A_{\mathrm{d}}-\overline{A_{\mathrm{d}}}=\sqrt{\frac{k_{\mathrm{B}} T \overline{A_{\mathrm{d}}}}{\kappa}}
$$

Considering $\kappa=0.1 \mathrm{~N} \mathrm{~m}^{-1}$, eqn (6) leads to a fluctuation of $1 \%$ in the domain area for domains of $10 \mathrm{~nm}$ radius and about $3 \%$ for domains of $4 \mathrm{~nm}$ radius, meaning that thermodynamic fluctuations would not lead to the annihilation of nano-sized domains in monolayers independent of the value of $\lambda$, provided that their radius is larger than $r_{\mathrm{c}}$, and thus, smaller domains probably coexist with the observed micron-sized domains but are not detected by optical microscopy.

\section{Conclusions}

In this work, the line tension in monolayers with liquid-liquid phase coexistence was determined in the presence of low amounts of molecules that may act as linactants. In most cases, a line tension of about $1 \mathrm{pN}$ was determined and of the four putative linactants tested, only one affected the line tension of the liquid ordered domains.

In the case of the fluorescent probes, while RhoPEegg did not affect the line tension, DiIC18 decreased it. Why RhoPEegg and DiIC18 affect the line tension differently is a question that cannot be completely answered at this stage. From the chemical structure (Fig. 2), we can hypothesize that DiIC18 would induce more disorder in the monolayer structure than RhoPEegg, since the fluorescent moiety of DiIC18 is bulkier and is closer to the hydrocarbon chains than that of RhoPEegg. This may be the reason for the accumulation of DiIC18 (and not of RhoPEegg) at the domain borders, which are already disordered regions. ${ }^{31-37}$

In relation to hybrid lipids, the constancy in the line tension of both mixtures, DLPC/dchol and DPPC/dchol, when POPC was added, indicates that this hybrid lipid does not act as a linactant in these monolayers, suggesting that the presence of a saturated 
and an unsaturated chain in a lipid may be a required condition but is not sufficient for a molecule to decrease the line tension in a monolayer system. This appears to be the case also for molecules with different chain lengths, since PLPC did not affect the line tension of monolayers composed of DLPC and dchol. For both types of molecules, we suggest that in spite of the favorable interactions that would generate at the interface in the presence of these molecules, the decrease in energy gained with this arrangement is not enough to pay the entropy cost for such ordered molecular distribution.

Regarding the membrane texture, it appears that domain size distribution in monolayers with liquid-ordered domains is in general a non-equilibrium property, not directly related to line tension. Additionally, thermodynamic analysis indicates that nano-sized domains in monolayers may be present, and they would be stable from this viewpoint independent of the value of $\lambda$, provided that their radius is larger than $r_{\mathrm{c}}$. Clearly, this analysis cannot be extrapolated to nano-sized domains in cell membranes, since these are very complex bilayer systems with a lot of different chemical species, the local composition of which continuously changes. However, regarding the chemical composition, a monolayer system with a high amount of different lipid species was previously studied, and line tension values in the same range of those found here were obtained. ${ }^{39}$ Yet, a similar analysis in a bilayer system would be required in order to better comprehend line tension and its regulation in cell membranes.

\section{Acknowledgements}

This work was supported by SECyT-UNC (05/C636), CONICET (PIP) and FONCYT (Program BID 0770) Argentina. N.W. is a Career Investigator and A.M. is a fellow from CONICET.

\section{References}

1 H. M. McConnell, Annu. Rev. Phys. Chem., 1991, 42, 171.

2 U. Bernchou, J. H. Ipsen and A. C. Simonsen, J. Phys. Chem. B, 2009, 113, 7170.

3 A. Gutierrez-Campos, G. Diaz-Leines and R. Castillo, J. Phys. Chem. B, 2010, 114, 5034.

4 M. Karttunen, M. P. Haataja, M. Saily, I. Vattulainen and J. M. Holopainen, Langmuir, 2009, 25, 4595.

5 C. W. McConlogue and T. K. Vanderlick, Langmuir, 1999, 15, 234.

6 S. Wurlitzer, T. M. Fischer and H. Schmiedel, J. Chem. Phys., 2002, 116, 10877.

7 F. Vega Mercado, B. Maggio and N. Wilke, Chem. Phys. Lipids, 2012, 165, 232.

8 A. J. Garcia-Saez, S. Chiantia and P. Schwille, J. Biol. Chem., 2007, 282, 33537.

9 F. Tokumasu, A. J. Jin, G. W. Feigenson and J. A. Dvorak, Biophys. J., 2003, 84, 2609.

10 A. Mangiarotti, B. Caruso and N. Wilke, Biochim. Biophys. Acta, 2014, 1838, 1823.

11 I. Sriram and D. K. Schwartz, Surf. Sci. Rep., 2012, 67, 143.

12 P. Muller and F. Gallet, Phys. Rev. Lett., 1991, 67, 1106.
13 D. J. Benvegnu and H. M. McConnell, J. Phys. Chem., 1993, 97, 6686.

14 M. Edidin, Annu. Rev. Biophys. Biomol. Struct., 2003, 32, 257.

15 J. F. Hancock, Nat. Rev. Mol. Cell Biol., 2006, 7, 456.

16 R. Brewster, P. A. Pincus and S. A. Safran, Biophys. J., 2009, 97, 1087.

17 S. Trabelsi, S. Zhang, T. R. Lee and D. K. Schwartz, Phys. Rev. Lett., 2008, 100, 037802.

18 R. B. Gennis, Biomembranes: Molecular Structure and Function, Springer-Verlag, New York, 1989.

19 B. Palmieri and S. A. Safran, Langmuir, 2013, 29, 5246.

20 L. V. Schafer and S. J. Marrink, Biophys. J., 2010, 99, L91.

21 T. Yamamoto and S. A. Safran, Soft Matter, 2011, 7, 7021.

22 C. M. Rosetti, B. Maggio and N. Wilke, Biochim. Biophys. Acta, 2010, 1798, 498.

23 A. A. Bischof and N. Wilke, Chem. Phys. Lipids, 2012, 165, 737.

24 D. W. Lee, Y. Min, P. Dhar, A. Ramachandran, J. N. Israelachvili and J. A. Zasadzinski, Proc. Natl. Acad. Sci. U. S. A., 2011, 108, 9425.

25 P. I. Kuzmin, S. A. Akimov, Y. A. Chizmadzhev, J. Zimmerberg and F. S. Cohen, Biophys. J., 2005, 88, 1120.

26 E. Hassan-Zadeh, E. Baykal-Caglar, M. Alwarawrah and J. Huang, Langmuir, 2014, 30, 1361.

27 N. Wilke and B. Maggio, Biophys. Chem., 2006, 122, 36.

28 N. Wilke, S. A. Dassie, E. P. M. Leiva and B. Maggio, Langmuir, 2006, 22, 9664.

29 S. Wurlitzer, P. Steffen and T. M. Fischer, J. Chem. Phys, 2000, 112, 5915.

30 T. M. Okogoni and H. M. McConnell, Biophys. J., 2004, 86, 880.

31 L. Cruzeiro-Hansson and O. G. Mouritsen, Biochim. Biophys. Acta, 1988, 944, 63.

32 G. Baneyx and V. Vogel, Proc. Natl. Acad. Sci. U. S. A., 1999, 26, 12518.

33 H. Haas and H. Mowald, Thin Solid Films, 1980, 180, 101.

34 M. L. F. Ruano, K. Nag, L. Worthman, C. Casals, J. Perez-Gil and K. M. W. Keough, Biophys. J., 1998, 1101.

35 C. I. N. Ayudhya, V. Prachayasittikul and H. Galla, Eur. Biophys. J., 2004, 33, 552.

36 R. R. Netz, D. Andelman and H. Orland, J. Phys. II, 1996, 6, 1023.

37 J. M. Crane and L. K. Tamm, Biophys. J., 2004, 86, 2965.

38 J. Israelachvili, Intermolecular and Surface Forces, Academic Press, London, 1991.

39 J. M. Pusterla, J. M. Hernandez, A. A. Bischof, N. Wilke and R. G. Oliveira, Myelin monolayers at the air/water interface: physical parameters of coexisting domains, in Libro de Resúmenes de la XLI Reunión Anual de la Sociedad Argentina de Biofísica, ed. R. Chehín, C. Ávila and C. Torres Bugeau, SAB-Sociedad Argentina de Biofísica, Buenos Aires, 2012, p. 197.

40 R. Brewster and S. A. Safran, Biophys. J., 2010, 98, L21.

41 P. Dhar, E. Eck, J. N. Israelachvili, D. W. Lee, Y. Min, A. Ramachandran, A. J. Waring and J. A. Zasadzinski, Biophys. J., 2012, 102, 56. 
42 S. Wurlitzer, P. Steffen, M. Wurlitzer, Z. Khattari and T. M. Fischer, J. Chem. Phys., 2000, 113, 3822.

43 H. M. McConnell, Proc. Natl. Acad. Sci. U. S. A., 1996, 93, 15001.

44 D. J. Benvegnu and H. M. McConnell, J. Phys. Chem., 1993, 97, 6686.

45 H. Mohwald, Annu. Rev. Phys. Chem., 1990, 41, 441.

46 D. Andelman, F. Brochard, C. M. Knobler and F. Rondelez, Micelles, Membranes, Microemulsions and Monolayers, ed. W. M. Gelbard, A. Ben-Shaul and D. A. Roux, Springer, New York, 1994, p. 559.

47 F. Vega Mercado, B. Maggio and N. Wilke, Chem. Phys. Lipids, 2012, 165, 232.

48 H. M. McConnell and R. De Koker, Langmuir, 1996, 12, 4897. 55 M. Blank, J. Phys. Chem., 1962, $66,1911$.
49 D. Gallez and H. M. McConnell, J. Phys. Chem. B, 2000, 104, 1657.

50 K. Y. Lee and H. M. McConnell, J. Phys. Chem., 1993, 97, 9532.

51 P. A. Rice and H. M. McConnell, Proc. Natl. Acad. Sci. U. S. A., 1989, 86, 6445.

52 C. D. Blanchette, W. C. Lin, C. A. Orme, T. V. Ratto and M. L. Longo, Biophys. J., 2008, 94, 2691.

53 F. Tokumasu, A. J. Jin, G. W. Feigenson and J. A. Dvorak, Biophys. J., 2003, 84, 2609.

54 F. A. Heberle, M. Doktorova, S. Lin Goh, R. F. Standaert, J. Katsaras and G. W. Feigenson, J. Am. Chem. Soc., 2013, 135, 14932 